Check for updates

Cite this: RSC Adv., 2017, 7, 55141

Received 6th October 2017

Accepted 16th November 2017

DOI: $10.1039 / \mathrm{c} 7 \mathrm{ra11006e}$

rsc.li/rsc-advances

\section{Rapid and sensitive detection of Salmonella enteritidis by a pre-concentrated immunochromatographic assay in a large-volume sample system $\dagger$}

\author{
Miao-Lin Duan, (DD a Yan-Mei Huang, ${ }^{c}$ Song-Song Wu, ${ }^{a}$ Guo-Qiang Li, ${ }^{a}$ \\ Shu-Ying Wang, ${ }^{a}$ Ming-Hui Chen, ${ }^{a}$ Chun Wang, ${ }^{a}$ Dao-Feng Liu, ${ }^{* b}$ Cheng-Wei Liu ${ }^{* b}$ \\ and Wei-Hua Lai*a
}

\begin{abstract}
A pre-concentrated immunochromatographic assay for Salmonella enteritidis (S. enteritidis) detection was developed based on the unique optical and magnetic properties of magnetic nanoparticles (MNPs). The target was easily concentrated from a large volume of sample by using immune MNPS (IMNPS) with superparamagnetism. The results could be directly distinguished using colored IMNPs on test strip. A target elution step was not required in the proposed method. The key parameters, including coupling $\mathrm{pH}$, blocking agents, and amount of IMNPS on the test strip, were optimized. Colloidal gold-based immunochromatographic assay (CG-ICA), MNP-based immunochromatographic assay (MNP-ICA), and MNP-ICA for a large-volume sample (large-volume MNP-ICA) were compared. Under optimal conditions, the sensitivity of large-volume MNP-ICA was $1.95 \times 10^{5} \mathrm{CFU} \mathrm{mL}{ }^{-1}$, which was 10 times higher than that of MNP-ICA and 1000 times higher than that of CG-ICA, owing to the preconcentration from a large sample volume. Moreover, the antibody consumption of large-volume MNPICA was lower than that of conventional CG-ICA. The entire detection time of the proposed method was only $30 \mathrm{~min}$. In general, the proposed method may be considered as a rapid, sensitive, and simple screening tool for on-site detection of $S$. enteritidis as well as other pathogens.
\end{abstract}

\section{Introduction}

Salmonella is the most common and primary cause of food contamination in many countries. ${ }^{1,2}$ Approximately 93.8 million cases of Salmonella infection and 155000 associated deaths occur each year. ${ }^{3}$ In Salmonella species, Salmonella enterica subsp. enterica serovar Enteritidis (S. enteritidis) is the main pathogen, infection of which involves typical symptoms of fever, diarrhea, vomiting, and acute gastroenteritis. ${ }^{4,5}$ As one of the most important causes of food-borne illness worldwide, among Salmonella serovars, the proportion of $S$. enteritidis isolated from humans was $43.5 \%$ in the North American and Oceania (Australia and New Zealand) regions. ${ }^{6}$ Traditional separation identification method, including pre-enrichment, selective

${ }^{a}$ State Key Laboratory of Food Science and Technology, Nanchang University, 235, Nanjing East Road, Nanchang 330047, China. E-mail: talktolaiwh@163.com ; Fax: +8679188333708; Tel: +8613879178802

${ }^{b}$ Institute for Nutrition and Food Safety, Jiangxi Province Centre for Disease Control and Prevention, 555, Beijing East Road, Nanchang 330029, China. E-mail: defoelau@163.com; liuchengwei718@sina.com

'Jiangxi YeLi Medical Device Co., Ltd, 2799, Tianxiang Road, Nanchang 330008, China $\dagger$ Electronic supplementary information (ESI) available. See DOI: 10.1039/c7ra11006e enrichment, isolated culture, biochemical identification, and serological identification, is used as the "gold standard" for detecting $S$. enteritidis in food samples. However, it takes 5-7 days to identify the target bacterium, and isolation and culture are also time-consuming and laborious. To address these issues, several effective methods for the detection of $S$. enteritidis have been developed, including enzyme-linked immunosorbent assay $^{7}$ (ELISA), electrochemical method, ${ }^{8}$ and polymerase chain reaction-based method. ${ }^{9}$ These detection methods have the advantages of high sensitivity and high specificity. ${ }^{10-12}$ However, these methods require trained personnel, complex procedure, or expensive instruments. To a certain degree, such drawbacks limit these methods from application in on-site detection.

Immunochromatographic assay (ICA) is a widely used technology in the field of rapid detection. ${ }^{13-16}$ Traditional colloidal gold-based immunochromatographic assay (CG-ICA) has four advantages: short period of time required to acquire test results, user-friendly format, and long-term stability over a wide range of climates, and relatively inexpensive production. However, CG-ICA shows limited detection sensitivity. ${ }^{17,18}$

Immunomagnetic separation has been a useful technology to concentrate and separate target pathogens from complex 
food samples. ${ }^{19-22}$ In recent years, immunomagnetic separation was also coupled with ICA to improve the detection sensitivity and specificity. For example, Cui et al. ${ }^{23}$ established a method combined immunomagnetic separation with CG-ICA to isolate and detect Escherichia coli O157:H7. Zhang et al. ${ }^{\mathbf{1 6}}$ reported a method for the detection of Enterobacter cloacae by immunomagnetic separation and a CG-ICA. The sensitivity of the method was 10 times higher than that of direct detection with ICA. Li et al. $^{\mathbf{2 4}}$ developed a rapid method to detect Listeria monocytogenes using fluorescence immunochromatographic assay combined with immunomagnetic separation and 40-fold improvement of detection limit was achieved compared with fluorescence immunochromatographic assay. In their previous work, the optical property of MNPs was underutilized. Target bacteria need to be eluted and then detected by an additional ICA. The elution step complicated the detection procedure and probably resulted in the loss of targets.

In this study, a MNP-based pre-concentrated ICA was developed for rapid and sensitive detection of $S$. enteritidis (largevolume MNP-ICA). A large-volume of sample ( $8 \mathrm{~mL}$ ) was used in this proposed method and concentrated to a small volume for $S$. enteritidis detection. IMNPs were used not only to capture $S$. enteritidis from large-volume sample but also to act as a colored signal label based on its optical property. Target elution step was omitted and the loss of target was avoided. The sensitivity of the proposed method was obviously improved with simplified experimental procedure.

\section{Materials and methods}

\subsection{Materials}

Reagents. Active $N$-hydroxysuccinimide esterified MNPs (mean diameter $150 \mathrm{~nm}$ ) were obtained from Enriching Biotechnology Co., Ltd. (LOT. Elut-P012, Shanghai, China). Anti-S. enteritidis polyclonal antibody Q1, which was used as the detection and capture antibody, was prepared by our laboratory. Donkey anti-rabbit antibody was obtained from Beijing ZSGBBio Co., Ltd. (Beijing, China). Bovine Serum Albumin (BSA) was purchased from Amresco (Washington, USA). 2-Morpholinoethanesulfonic acid (MES) was obtained from Alfa Aesar (Heysham, USA). Hydrogen tetrachloroaurate(III) trihydrate $\left(\mathrm{HAuCl}_{4} \cdot 3 \mathrm{H}_{2} \mathrm{O}\right)$, sodium citrate $\left(\mathrm{C}_{6} \mathrm{H}_{5} \mathrm{Na}_{3} \mathrm{O}_{7} \cdot 2 \mathrm{H}_{2} \mathrm{O}\right)$, casein sodium, D-glucosamine, and ethanolamine were obtained from Sigma-Aldrich Chemical Co. (St. Louis, MO, USA). Nitrocellulose membrane was obtained from Millipore (Bendford, MA, USA). The sample pad, conjugate pad, absorbent pad, and PVC plate were purchased from Shanghai Jinbiao Biotechnology Co., Ltd. (Shanghai, China). Peptone and yeast extract powder were purchased from Oxoid (Basingstoke, England). Bicinchoninic acid kit for protein determination (BCA) was obtained from Thermo Fisher Scientific Co., Ltd. (Massachusetts, USA). All other chemicals and reagents were of analytical reagent grade and were obtained from Aladdin (Shanghai, China). Low fat milk was bought from a local grocery store.

Equipment. Optical signal strip reader was purchased from Shanghai Huguo Science Instrument Co., Ltd. (Shanghai,
China). BioDot XYZ platform and other strip manufacture equipment were obtained from BioDot (Irvine, CA).

Bacterial strains. Listeria welshimeri (ATCC 35897), Candida albicans (ATCC 10231), Bacillus subtilis (BD 168), Proteusbacillus vulgaris (CMCC 49027), Shigella flexneri (CMCC 2457), Enterobacter sakazakii (CMCC 45407), Pseudomonas aeruginosa (CMCC 11997), Escherichia coli 0157:H7 (ATCC 43888), Micrococcus luteus (CMCC 28001) and Salmonella enteritidis (ATCC 13076) were used in this work. All strains were preserved in our laboratory.

\subsection{Preparation of probes}

A total of $100 \mu \mathrm{L}$ of active $N$-hydroxysuccinimide esterified MNPs $\left(10 \mathrm{mg} \mathrm{mL}^{-1}\right.$ ) with the diameter of $150 \mathrm{~nm}$ was added into a centrifuge tube. After washing with MES buffer solution twice to remove the excess chemical reagents, $1 \mathrm{~mL}$ of $\mathrm{pH}$ adjusted purified anti-S. enteritidis polyclonal antibody (Q1) solution $\left(60 \mu \mathrm{g} \mathrm{mL}{ }^{-1}\right)$ was added to the centrifuge tube. The mixture was reacted at room temperature for $4 \mathrm{~h}$. After magnetic separation, the supernatant was used for subsequent calculation of coupling rate. Afterward, $1 \mathrm{~mL}$ of $3 \%$ BSA (w/v) was added in the centrifuge tube. The antibody-conjugated MNPs were then blocked overnight at $4{ }^{\circ} \mathrm{C}$. The supernatant was removed in the magnetic field, and the precipitate was resuspended with $1 \mathrm{~mL}$ of phosphate buffered saline solution containing $3 \%$ BSA and $0.1 \%$ Tween $20(\mathrm{v} / \mathrm{v})(\mathrm{pH} 8.5)$ and then stored at $4{ }^{\circ} \mathrm{C}$.

The colloidal gold with an average diameter of $30 \mathrm{~nm}$ was produced by reduction of gold chloride trihydrate with $1 \%$ trisodium citrate as described previously ${ }^{25}$ and immune colloidal gold (ICG) was prepared according to the method of Peng. ${ }^{26}$

\subsection{Calculation of coupling rate and capture efficiency}

Coupling rate is defined as the percentage fraction of the antibody bound onto the surface of the MNPs. The concentration of anti-S. enteritidis polyclonal antibody Q1 left in the supernatant was calculated by BCA assay.

The BCA working reagent was prepared by mixing the solutions A, B, and C at $25: 24: 1$ proportion. Serial dilutions from the standard concentration were prepared from the BSA and assayed alongside the unknown(s) before the concentration of each unknown is determined based on the standard curve. In brief, $150 \mu \mathrm{L}$ of standard BSA solution and the supernatant reserved from the preparation of IMNPs was added into an ELISA well. Afterward, $150 \mu \mathrm{L}$ of the working solution was added into the well, and the mixture was incubated at $37^{\circ} \mathrm{C}$ for $2 \mathrm{~h}$ in dark. Optical density (OD) at $560 \mathrm{~nm}$ was measured using a Microplate Reader, and the result was calculated according to the standard curve drawn. Coupling rate was calculated using the following formula:

$$
\text { Coupling rate }(\%)=\left(1-A / A_{0}\right) \times 100 \%
$$

where $A_{0}$ is the original amount of antibody (added in total) and $A$ is the amount of antibody in the supernatant. 
The number of $S$. enteritidis in the supernatant after capture was measured using the plate counting method. Capture efficiency was calculated using the following formula:

$$
\text { Capture efficiency }(\%)=\left(1-N / N_{0}\right) \times 100 \%
$$

where $N_{0}$ (CFU) is the original number of $S$. enteritidis (added in total) and $N$ (CFU) is the number of $S$. enteritidis in the supernatant.

\subsection{Preparation of test strip}

Anti-S. enteritidis polyclonal antibody Q1 (1.5 $\left.\mathrm{mg} \mathrm{mL}^{-1}\right)$ and donkey anti-rabbit antibody $\left(0.5 \mathrm{mg} \mathrm{mL}^{-1}\right)$ were sprayed as test line ( $\mathrm{T}$ line) and control line (C line) on nitrocellulose membrane, respectively. Afterward, the nitrocellulose membrane was dried at $37{ }^{\circ} \mathrm{C}$ for $2 \mathrm{~h}$ in a vacuum drying chamber. The sample pad was immersed in $0.1 \mathrm{M}$ phosphate buffer containing $1 \%$ BSA (w/v), 0.5\% Tween 20 (v/v), and 0.05\% $\mathrm{NaN}_{3}(\mathrm{w} / \mathrm{v})$, and the conjugate pad was immersed in $0.1 \mathrm{M}$ phosphate buffer containing $0.25 \%$ Tween $20(\mathrm{v} / \mathrm{v})$ and $2 \%$ glucose $(\mathrm{w} / \mathrm{v})$. The sample pad and conjugate pad were dried at $37{ }^{\circ} \mathrm{C}$ overnight in a vacuum drying chamber. The prepared nitrocellulose membrane, conjugate pad, sample pad, and absorption pad were then assembled as the test strip.

\subsection{Optimizations of key parameters}

The coupling $\mathrm{pH}$ of $4,5,6,7,8,9$, and 10 were explored in the conjugation of MNPs and anti-S. enteritidis polyclonal antibody Q1. The coupling rate and capture efficiency were used to evaluate the influence of coupling $\mathrm{pH}$ on the IMNPs conjugation.

Four commonly used blocking agents in the preparation of IMNPs, including $2 \%$ ethanolamine $(\mathrm{w} / \mathrm{v})$, D-glucosamine $\left(1 \mathrm{mg} \mathrm{mL}{ }^{-1}\right), 3 \% \mathrm{BSA}$, and $3 \%$ casein sodium $(\mathrm{w} / \mathrm{v})$ were tested and evaluated in the study. A total of $4 \mu \mathrm{L}$ of IMNPs blocked with four blocking agent solutions were added to the strips. A blocking agent was considered ideal in the presence and absence of a colored signal in the $\mathrm{C}$ line and the $\mathrm{T}$ line, respectively.

To determine the appropriate additive amount of IMNPs on the strip, different additive amounts of IMNPs $(2,4,8,16$, and $24 \mu \mathrm{g}$, all in $100 \mu \mathrm{L}$ ) were tested. The mixture solution was added into the strip, and the background color and color signal of $\mathrm{T}$ and $\mathrm{C}$ lines of the strip were observed and evaluated after $15 \mathrm{~min}$.

\subsection{Immunological kinetics analysis of IMNPs and ICG}

Outer membrane protein antigen was extracted $\left(0.4 \mathrm{mg} \mathrm{mL} \mathrm{mL}^{-1}\right)$ from $S$. enteritidis and was sprayed on the $\mathrm{C}$ line to build a strip for running this comparison. A total of $6 \mu \mathrm{g}$ of IMNPs or $6 \mu \mathrm{L}$ of ICG was added to the strip. The optical signals on the $\mathrm{T}$ line were repeatedly read every $15 \mathrm{~s}$ by using an optical signal strip reader. The kinetic curves were established by plotting the signal intensity of $\mathrm{T}$ line against time.

\subsection{Sensitivity test of three ICAs}

Under optimized conditions, the procedure for large-volume MNP-ICA was conducted as follows. A total of $50 \mu \mathrm{g}$ of IMNPs was added into $8 \mathrm{~mL}$ of $S$. enteritidis-enriched culture broth at room temperature for $20 \mathrm{~min}$. After magnetic separation, the precipitate was resuspended in $625 \mu \mathrm{L}$ of Luria-Bertani broth $(8 \mu \mathrm{g}$ IMNPs per $100 \mu \mathrm{L})$ containing $0.3 \%$ BSA (w/v) and $0.01 \%$ Tween $20(\mathrm{v} / \mathrm{v})$, and $100 \mu \mathrm{L}$ of the resuspended solution was applied to the test strip directly without elution step. After $10 \mathrm{~min}$, the results were read with the naked eye.

For comparison, the CG-ICA and MNP-ICA were developed and optimized according to previous work. ${ }^{19,27}$ The procedure of CG-ICA and MNP-ICA were conducted as follows. A total of $8 \mu \mathrm{L}$ of ICG or $8 \mu \mathrm{g}$ of IMNPs was added in $100 \mu \mathrm{L}$ of a series of $S$. enteritidis dilutions $\left(1.95 \times 10^{3}\right.$ to $\left.1.95 \times 10^{8} \mathrm{CFU} \mathrm{mL}^{-1}\right)$, and $100 \mu \mathrm{L}$ of the mixture was transferred into the test strip. After $15 \mathrm{~min}$, the results were read with the naked eye.

\subsection{Specificity test}

S. enteritidis at a concentration of $10^{6} \mathrm{CFU} \mathrm{mL}^{-1}$ and nine non$S$. enteritidis strains at a concentration of $10^{6} \mathrm{CFU} \mathrm{mL}^{-1}$ were tested to evaluate the specificity of the proposed method.

\subsection{Detection of $S$. enteritidis in food sample}

Low fat milk ( $3 \%$ milk fat) samples were spiked with diluted cultures of $S$. enteritidis with final concentrations of $1.95 \times 10^{8}$, $1.95 \times 10^{7}, 1.95 \times 10^{6}, 1.95 \times 10^{5}, 1.95 \times 10^{4}$, and $1.95 \times 10^{3}$ CFU mL ${ }^{-1}$. Large-volume MNP-ICA was applied in this test and the results were obtained after 15 min (Fig. 1)

\section{Results and discussion}

\subsection{Effects of coupling $\mathrm{pH}$ on IMNPs}

The coupling $\mathrm{pH}$ is a key parameter in the conjugation of MNPs and antibody. At different $\mathrm{pH}$ conditions, the exposed domain that reacts with MNPs and the activity of antibody are both affected..$^{28}$ As shown in Fig. 2 and Table 1, as pH decreased from 10 to 5 , both coupling rate and capture efficiency seemed to be on a slight upward trend. The amino groups on the antibody are hypothesized as more likely to be positively charged in an acidic environment. Therefore, the conjugation between MNPs and antibody seemed to be easier at more acidic environments. However, when the $\mathrm{pH}$ was too low $(\mathrm{pH}=4)$, the active esters tended to be more stable, that is, most parts of the ester group on the surface of MNPs become inactive, and MNPs and antibody became hard to conjugate. At $\mathrm{pH}$, both the coupling rate and capture efficiency reached their highest value. Thus, $\mathrm{pH} 5$ was selected as the appropriate coupling $\mathrm{pH}$ for the conjugation.

\subsection{Effects of blocking agent on IMNPs}

Blocking is an important step in the process of IMNPs preparation. An ideal blocking agent can encapsulate IMNPs to prevent them from binding to other substances in the 


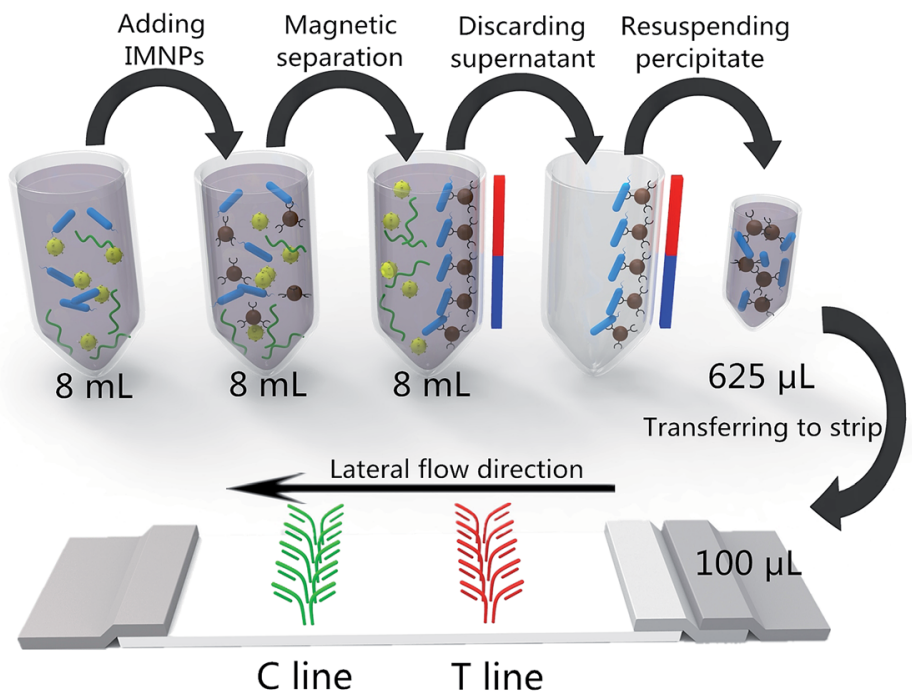

\section{S. enteritidis Other bacteria $\sim$ Other bacteria}
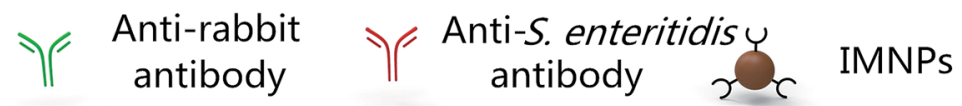

Fig. 1 Schematic of large-volume MNP-ICA.

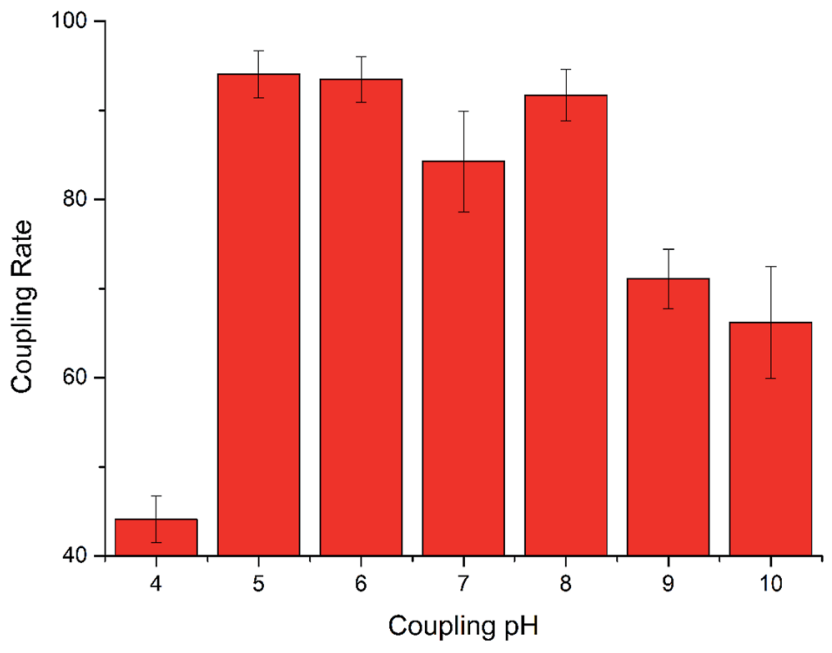

Fig. 2 The effect of $\mathrm{pH}$ value on coupling rate.

chromatographic system and can quench the active group that was not consumed by the antibody in the coupling process.

Four different commonly used blocking agents (ethanolamine, D-glucosamine, BSA, and casein sodium) were compared. In the absence of a blocking agent, the IMNPs were easily bound to the antibodies on the $\mathrm{T}$ line because of many

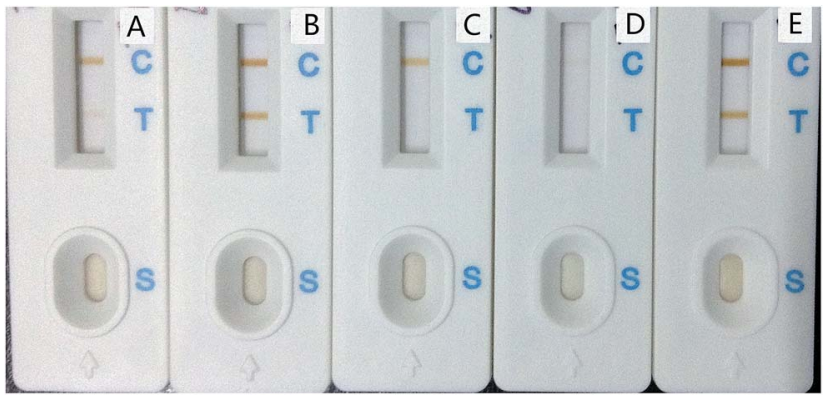

Fig. 3 Comparison of different blockers. (A) 2\% Ethanolamine (v/v); (B) $1 \mathrm{mg} \mathrm{mL} \mathrm{m}^{-1}$-glucosamine; (C) $3 \% \mathrm{BSA}(\mathrm{w} / \mathrm{v})$; (D) $3 \%$ casein sodium $(\mathrm{w} / \mathrm{v})$; (E) without blocking.

unquenched active sites (Fig. 3E). Moreover, blocking IMNPs by ethanolamine and D-glucosamine also showed undesirable $\mathrm{T}$ line on the strips (Fig. 3A and B), which was hypothesized to be due to a number of hydroxyl and other charged groups introduced, which brought about nonspecific binding even though the active ester was quenched. When IMNPs were blocked with casein sodium solution (Fig. 3D), weak color signal on both $\mathrm{T}$ line and $\mathrm{C}$ line appeared, which was speculated to be due to immune reaction inhibition by casein sodium. However, when IMNPs were blocked by BSA solution, the color signal appeared

Table 1 Capture efficiency at different coupling pH

\begin{tabular}{|c|c|c|c|c|c|c|c|}
\hline Coupling pH & 4 & 5 & 6 & 7 & 8 & 9 & 10 \\
\hline Capture efficiency (\%) & $23.8 \pm 4.8$ & $99.3 \pm 0.2$ & $97.6 \pm 0.6$ & $95.9 \pm 1.0$ & $96.3 \pm 1.2$ & $96.1 \pm 1.2$ & $92.2 \pm 0.5$ \\
\hline
\end{tabular}


in $\mathrm{C}$ line without nonspecific binding on the $\mathrm{T}$ line as shown in Fig. 3C. Thus, BSA was chosen as the optimal blocking agent.

\subsection{Effects of additive amount of IMNPs}

When IMNPs were added to the test in excess, portions of IMNPs were deposited on the nitrocellulose membrane, resulting in a brown background color on the membrane. By contrast, when the IMNPs were added in too low amounts, the colored signal in the $\mathrm{C}$ line was weak and the detection sensitivity also decreased. To have an appropriate signal color and a weak background color, the additive amounts of IMNPs should be explored.

As shown in Fig. 4, the colored signal on the $\mathrm{C}$ line increased in intensity as the additive amount of IMNPs was increased. However, excessive IMNPs have obvious impact on background color on the whole nitrocellulose membrane. As $16 \mu \mathrm{g}$ and $24 \mu \mathrm{g}$ of IMNPs were added, a brown background on the strip was obvious.

An additive amount of $8 \mu \mathrm{g}$ permitted a strong colored signal on the $\mathrm{C}$ line and an absence of background color on the nitrocellulose membrane. Thus, $8 \mu \mathrm{g}$ of IMNPs was selected as the appropriate additive amount.

\subsection{Immunological kinetics analysis of IMNPs and ICG}

To further explore the reasons behind the improvement in sensitivity, the dynamic properties between immunochromatography of MNPs and CG were compared. As shown in Fig. 5, using the same antibody-antigen system, the peak grayscale intensity of MNPs (917.4) was about 1.5 times higher than that of CG (607.4). Moreover, the antibody concentration used for MNP-ICA was $0.36 \mu \mathrm{g}$, whereas that for the CG-ICA was $3.6 \mu \mathrm{g}$. Compared with CG, 1.5 times signal intensity was produced by MNPs, with a 1/10 antibody consumption. As label in immunochromatographic assay, MNPs produce stronger signal intensity, demonstrating higher sensitivity than the traditional and widely used CG. Moreover, the consumption of antibody could be greatly reduced when MNPs are used as the label, indicating its industrial and economical value.

\subsection{Results of sensitivity test of three ICAs}

As shown in Fig. 6, the sensitivity of traditional CG-ICA and MNPICA were $1.95 \times 10^{8}$ and $1.95 \times 10^{6}$, respectively. However, when

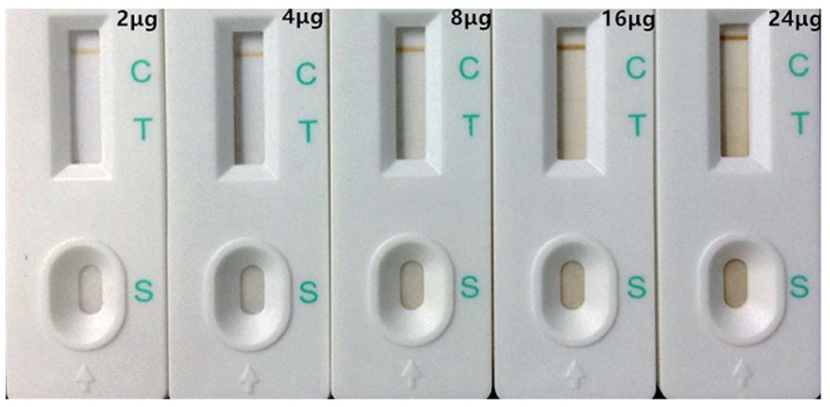

Fig. 4 Comparison of different additive amount of IMNPs.

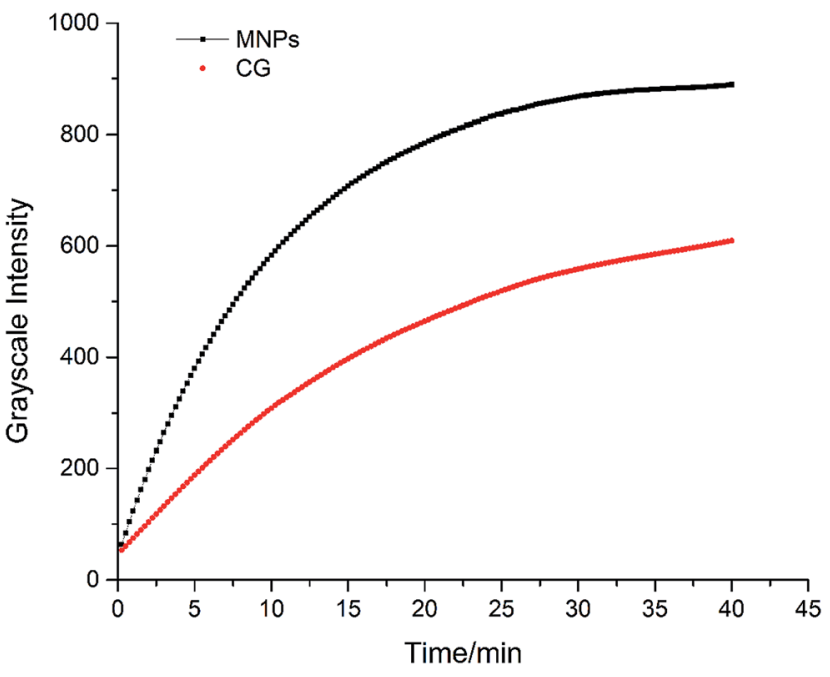

Fig. 5 Immunological kinetics of MNPs and CG.

the concentration of $S$. enteritidis was at $1.95 \times 10^{5} \mathrm{CFU} \mathrm{mL}^{-1}$, a visible $\mathrm{T}$ line was produced in the proposed large-volume MNPICA.

Using the same antibody, the sensitivity of large-volume MNP-ICA was 10 times higher than that of MNP-ICA. In largevolume MNP-ICA, $50 \mu \mathrm{g}$ of IMNPs were added into $8 \mathrm{~mL}$ of sample solution. After magnetic separation, the sample was
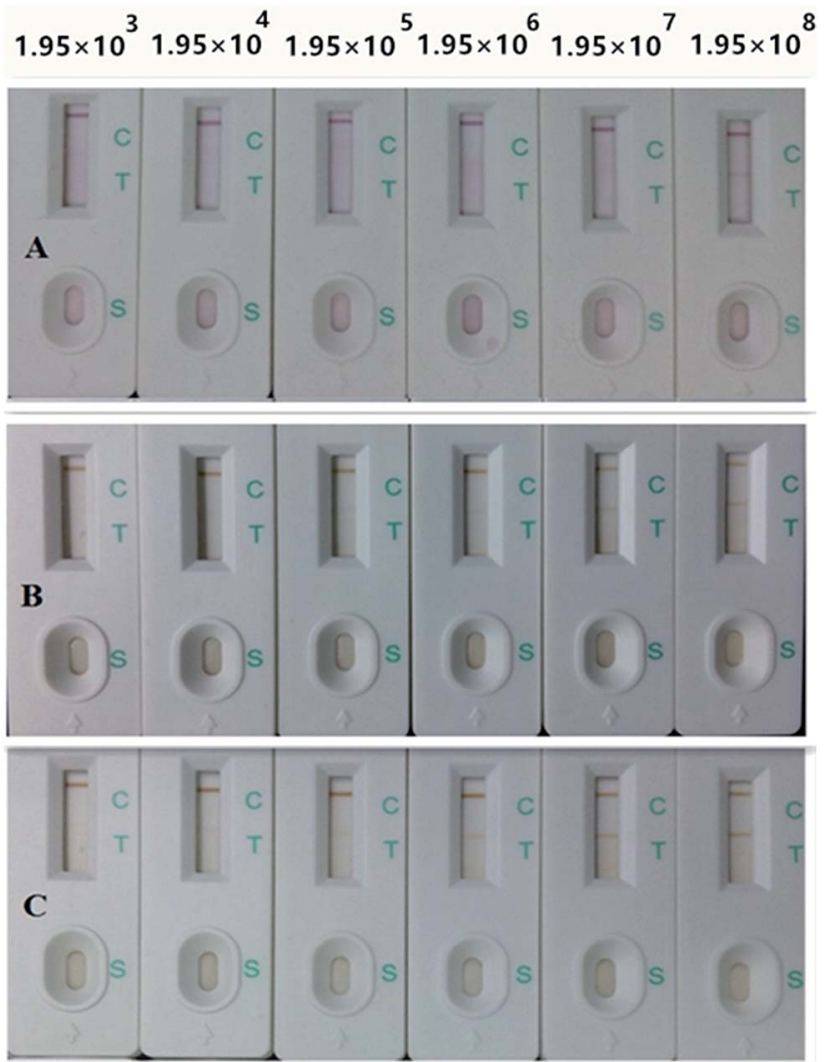

Fig. 6 Sensitivity of CG-ICA (A), MNP-ICA (B), and large-volume MNPICA (C) for detection of $S$. enteritidis. 
Table 2 Comparisons among CG-ICA, MNP-ICA, and large-volume MNP-ICA for the detection of S. enteritidis

\begin{tabular}{|c|c|c|c|c|c|}
\hline Methods & Additive amount of IMNPs/ICG & Antibody consumption & Sample volume & $\begin{array}{l}\text { Concentration } \\
\text { ratio }\end{array}$ & Sensitivity \\
\hline CG-ICA & $8.0 \mu \mathrm{L}$ per test & $4.8 \mu \mathrm{g}$ per test & $100.0 \mu \mathrm{L}$ & $1: 1$ & $1.95 \times 10^{8} \mathrm{CFU} \mathrm{mL}^{-1}$ \\
\hline Large-volume MNP-ICA & $50.0 \mu \mathrm{g}$ per test & $3.0 \mu \mathrm{g}$ per test & $8.0 \mathrm{~mL}$ & $1: 12.8$ & $1.95 \times 10^{5} \mathrm{CFU} \mathrm{mL}^{-1}$ \\
\hline
\end{tabular}

concentrated by 12.8 times. An MNP-based pre-concentrated immunochromatographic assay offers improved sensitivity due to the concentration of sample, as demonstrated by the similarity between the rate of sensitivity enhancement and the rate of concentration increase.

Moreover, compared with traditional CG-ICA, the sensitivity of the proposed large-volume MNP-ICA was 1000 times higher using the same antibody. We presume that the diameter of MNPs was larger than that of CG, thus the flow rate of IMNPs was slower than that of ICG, leading to a more sufficient immune reaction on $\mathrm{T}$ line.

Some parameters of the three ICAs are listed in Table 2. From the comparison between CG-ICA and MNP-ICA, the antibody consumption per test of MNP-ICA was only 1/10 of CG-ICA, whereas the sensitivity was 100 times higher than that of the latter, proving that as an optical label, IMNPs not only improved the detection sensitivity but also diminished antibody consumption.

Based on the unique optical and magnetic properties of MNPs, IMNPs not only improves the detection sensitivity but also offers great economic potential.

\subsection{Results of specificity test}

To examine the specificity of the large-volume MNP-ICA, crossreactivity during assay development must be tested. Nine nontarget strains were used to evaluate the specificity of the proposed method and no brown band appeared in the test line when each bacterium was added at concentration of $10^{6} \mathrm{CFU}$ $\mathrm{mL}^{-1}$ (Fig. S2 $\dagger$ ). This result indicated that the test strip has high specificity.

\subsection{Results of detection in food sample}

The detection results of $S$. enteritidis in low fat milk was shown in Fig. S3. $\dagger 1.95 \times 10^{5} \mathrm{CFU} \mathrm{mL}{ }^{-1}$ of $S$. enteritidis in milk could be detected with large-volume MNPs-ICA by naked eye. The sensitivity of the method with the low fat milk was basically consistent with that with Luria-Bertani broth $\left(1.95 \times 10^{5} \mathrm{CFU} \mathrm{mL}^{-1}\right)$. The results showed that this proposed method had little matrix effect.

\section{Conclusions}

A pre-concentrated immunochromatographic assay was developed for the rapid and sensitive detection of $S$. enteritidis in a large of volume sample based on the magnetic and optical properties of MNPs. The sensitivity of large-volume MNP-ICA was $1.95 \times 10^{5} \mathrm{CFU} \mathrm{mL}{ }^{-1}$, which was 10 times higher than that of MNP-ICA, and 1000 times higher than that of CG-ICA.

In the proposed method, the MNPs were used not only as a magnetic capturing probe to concentrate the target from a large-volume of sample but also as a colored signal label based on its optical property. No additional elution operation was required, thus target loss during elution was avoided. Furthermore, immunological kinetics comparison between MNP and CG showed that compared with CG, MNP produced much higher signal strength with less antibody consumption. MNP proved to be an ideal optical label in addition to being a sample pretreatment tool. Most importantly, the sensitivity of the proposed method was clearly improved considering the preconcentration of a large volume of sample $(8 \mathrm{~mL})$.

Considering these advantages, the proposed method is worthy to be applied for both on-site and in the laboratory, and it offers an important role in the field of food safety.

\section{Conflicts of interest}

There are no conflicts to declare.

\section{Acknowledgements}

This work was supported by the National Natural Science Foundation of China (no. 31772066), Young Scientist Fund of Jiangxi Province (2016BAB21418), Jiangxi Special Fund for Agroscientific Research in the Collaborative Innovation (JXXTCX201703-1), Jiangxi Agriculture Research System (JXARS03), and Science and Technology Program of Health and family planning commission of Jiangxi Province (20176011).

\section{Notes and references}

1 D. Zhu, Y. Yan, P. Lei, B. Shen, W. Cheng, H. Ju and S. Ding, Anal. Chim. Acta, 2014, 846, 44-50.

2 G. Muluneh and M. Kibret, Asian Pac. J. Trop. Dis., 2015, 5, 130-135.

3 S. E. Majowicz, J. Musto, E. Scallan, F. J. Angulo, M. Kirk, S. J. O'Brien, T. F. Jones, A. Fazil and R. M. Hoekstra, Clin. Infect. Dis., 2010, 50, 882-889.

4 C. H. Kuan, L. W. K. Lim, T. W. Ting, Y. Rukayadi, S. H. Ahmad, C. W. J. Wan Mohamed Radzi, T. Y. Thung, O. B. Ramzi, W. S. Chang, Y. Y. Loo, C. S. Kuan, S.-K. Yeo and S. Radu, Food Control, 2017, 80, 395-400.

5 G. Mellon, C. Delanoe, A. L. Roux, B. Heym, O. Dubourg, P. Hardy, B. Chevallier, C. Perronne, E. Rouveix and 
J. Salomon, Medicine and infectious diseases, 2017, 47, 389393.

6 R. S. Hendriksen, A. R. Vieira, S. Karlsmose, D. M. Lo Fo Wong, A. B. Jensen, H. C. Wegener and F. M. Aarestrup, Foodborne Pathog. Dis., 2011, 8, 887-900.

7 S. A. Mirhosseini, A. A. I. Fooladi, J. Amani and H. Sedighian, Braz. J. Microbiol., 2017, 48, 774-781.

8 X. Ma, Y. Jiang, F. Jia, Y. Yu, J. Chen and Z. Wang, J. Microbiol. Methods, 2014, 98, 94-98.

9 J. Y. Hyeon and X. Deng, Food Microbiol., 2017, 63, 111-116.

10 E. Galikowska, D. Kunikowska, E. Tokarska-Pietrzak, H. Dziadziuszko, J. M. Los, P. Golec, G. Wegrzyn and M. Los, Eur. J. Clin. Microbiol. Infect. Dis., 2011, 30, 10671073.

11 D. Lu, G. Pang and J. Xie, Biomed. Microdevices, 2017, 19, 12. 12 J. Chen, L. Zhang, G. C. Paoli, C. Shi, S. I. Tu and X. Shi, Int. J. Food Microbiol., 2010, 137, 168-174.

13 W. C. Mak, V. Beni and A. P. F. Turner, TrAC, Trends Anal. Chem., 2016, 79, 297-305.

14 M. Sajid, A.-N. Kawde and M. Daud, J. Saudi Chem. Soc., 2015, 19, 689-705.

15 S. H. Paek, S. H. Lee, J. H. Cho and Y. S. Kim, Methods, 2000, 22, 53-60.

16 X. Zhang, J. Zhou, C. Zhang, D. Zhang and X. Su, RSC Adv., 2016, 6, 1279-1287.
17 Q. Y. Xie, Y. H. Wu, Q. R. Xiong, H. Y. Xu, Y. H. Xiong, K. Liu, Y. Jin and W. H. Lai, Biosens. Bioelectron., 2014, 54, 262-265.

18 J. Li, H. Duan, P. Xu, X. Huang and Y. Xiong, RSC Adv., 2016, 6, 26178-26185.

19 Q. Xiong, X. Cui, J. K. Saini, D. Liu, S. Shan, Y. Jin and W. Lai, Food Control, 2014, 37, 41-45.

20 S. Shan, Z. Zhong, W. Lai, Y. Xiong, X. Cui and D. Liu, Food Control, 2014, 45, 138-142.

21 P. M. Fratamico, F. J. Schultz and R. L. Buchanan, Food Microbiol., 1992, 9, 105-113.

22 W.-B. Shim, J.-G. Choi, J.-Y. Kim, Z.-Y. Yang, K.-H. Lee, M.-G. Kim, S.-D. Ha, K.-S. Kim, K.-Y. Kim, C.-H. Kim, S. A. Eremin and D.-H. Chung, J. Food Prot., 2008, 71, 781789.

23 X. Cui, Q.-R. Xiong, Y.-H. Xiong, S. Shan and W.-H. Lai, Chin. J. Anal. Chem., 2013, 41, 1812-1816.

24 Q. Li, S. Zhang, Y. Cai, Y. Yang, F. Hu, X. Liu and X. He, Int. J. Food Sci. Technol., 2017, 52, 1559-1566.

25 C. Li, W. Luo, H. Xu, Q. Zhang, H. Xu, Z. P. Aguilar, W. Lai, H. Wei and Y. Xiong, Food Control, 2013, 34, 725-732.

26 T. Peng, W.-c. Yang, W.-H. Lai, Y.-H. Xiong, H. Wei and J. Zhang, Anal. Methods, 2014, 6, 7394-7398.

27 J.-Y. Wang, M.-H. Chen, Z.-C. Sheng, D.-F. Liu, S.-S. Wu and W.-H. Lai, RSC Adv., 2015, 5, 62300-62305.

28 Y. Chen, Y. Xin, H. Yang, L. Zhang, Y. Zhang, X. Xia, Y. Tong and W. Wang, Int. J. Biol. Macromol., 2013, 56, 6-13. 